\title{
Increased inflammatory cytokines and new collagen formation in cutaneous tuberculosis and sarcoidosis
}

\author{
Ben G Marshall, Arun Wangoo, H Terence Cook, Rory J Shaw
}

\begin{abstract}
Background - Interactions between mononuclear cells, vascular endothelium, fibroblasts, and cytokines during the inflammatory reaction within a granuloma have the potential to contribute to the pro-
\end{abstract} gression to fibrosis.

Methods - Biopsy specimens of six tuberculous and eight sarcoidosis skin lesions were examined by immunohistochemistry to seek evidence for the presence of inflammatory and fibrotic reactions in human granulomatous disease. Additionally, to understand how a $\mathbf{T}$ cell mediated delayed type hypersensitivity reaction - a component of chronic granulomatous inflammation - could progress to fibrosis, the human in vivo model of the cutaneous tuberculin Heaf reaction to purified protein derivative (PPD) was studied in a group of 48 subjects.

Results - Granulomas from tuberculous and sarcoidosis skin biopsy specimens were seen to contain cells with marked staining by antibodies to fibronectin, transforming growth factor $\beta$ (pan TGF$\beta)$, and type 1 procollagen (PCP-1). Accentuated staining of extracellular matrix was seen both in the granulomas and in the peri-granulomatous regions. Less prominent staining was observed using

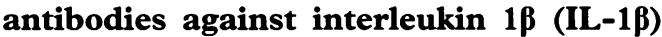
and $\alpha$-smooth muscle actin ( $\alpha$-SMA). Biopsies of Heaf reactions revealed cells staining for IL-1ß, tumour necrosis factor $\alpha$ (TNF- $\alpha)$, platelet derived growth factor $B$ (PDGF-B), and fibronectin which were detected as early as day 1 and persisted throughout the 14 day study period. Cells staining for PCP-1 increased to greatest abundance at day 14 . All these cytokines were present in low abundance in biopsy specimens from sites inoculated with saline only.

Conclusions - Evidence is provided that granulomas in tuberculosis and sarcoidosis behave as active centres of fibrogenesis. Using the Heaf model, the temporal relationship between the early appearance of cytokines and the later increase in the collagen precursor PCP-1 linked the immune mediated chronic inflammatory response with subsequent fibrosis and suggested that the tuberculin Heaf reaction will serve as a model for studying the early events of granuloma formation in patients with tuberculosis and sarcoidosis.

(Thorax 1996;51:1253-1261)

Keywords: type I procollagen, delayed type hypersensitivity reaction, tuberculin, immunohistochemistry, granuloma.

Granulomatous diseases such as sarcoidosis and tuberculosis commonly evolve towards irreversible lung scarring. ${ }^{1}$ In the skin tuberculosis (particularly the lupus vulgaris form) causes fibrosis. Scarring is less common in sarcoidosis of the skin, except in the papular and annular forms, ${ }^{2}$ although the immunohistology of skin granulomas in patients with sarcoidosis is similar to that of granulomas found in other organs such as lung and lymph nodes. $^{3}$

The inflammatory response in both diseases is characterised by $\mathrm{T}$ cell and macrophage activation, a delayed type hypersensitivity (DTH) response, and the presence of inflammatory cytokines. ${ }^{4}$ Thus, these diseases may represent models in which to understand the relationship between immune mediated inflammation and fibrosis, as well as serving as human disease models for other fibrotic lung diseases. ${ }^{5}$

Cytokines implicated in the progression from chronic inflammation to fibrosis include interleukin $1 \beta$ (IL-1 $\beta)^{67}$ and tumour necrosis factor $\alpha$ (TNF- $\alpha)$. The latter plays a central role in the evolution of pulmonary fibrosis ${ }^{89}$ as well as in granuloma formation and maintenance, both in the mouse ${ }^{10}$ and in humans. ${ }^{11}$ Both IL$1 \beta$ and TNF- $\alpha$ are considered the primary recruiters of cells in granuloma growth. ${ }^{12}$ Other cytokines such as transforming growth factor $\beta$ (TGF- $\beta$ ) and platelet derived growth factor (PDGF) are important regulators of tissue repair, but may result in unresolved inflammation and fibrosis when present in excess quantities. ${ }^{13-15}$ There is evidence implicating both $\mathrm{PDGF}^{1617}$ and TGF- $\beta^{1819}$ in the progression from chronic inflammation to fibrosis in tuberculosis and sarcoidosis.

The final common pathway in wound healing and granulomatous fibrotic reactions is the synthesis of collagen by fibroblasts, particularly the type $\mathrm{I}$ isoform, ${ }^{20}$ as well as other matrix proteins such as fibronectin..$^{21}$ Myofibroblasts are specialised fibroblasts with morphological and functional features of smooth muscle differentiation, one of a number of spindleshaped interstitial cells capable of actively producing matrix proteins and participating in the formation of granulation tissue. They are 
thought to be responsible for the production of connective tissue components which characterise fibrotic situations. ${ }^{22}$ Their hallmark is the production of $x$-smooth muscle actin which may be induced in some situations by TGF$\beta .^{23}$

In the present study we hypothesised that the chronic inflammatory response within cutaneous granulomas of tuberculosis and sarcoidosis is intimately associated with the progression to fibrosis. Biopsy specimens from cutaneous tuberculous and sarcoidosis lesions were examined by conventional immunohistochemical techniques to investigate the local production of these cytokines, fibrogenic growth factors, and matrix proteins. To dissect the local immune response and to examine the initial kinetics of a $T$ cell mediated inflammatory reaction we have applied the same techniques to the well established model of the cutaneous tuberculin Heaf test, a classical and fully defined example of a delayed type hypersensitivity reaction, ${ }^{24-26}$ since it may serve as a model of the early immunopathological response in tuberculosis and sarcoidosis.

\section{Methods \\ PATIENTS \\ Granulomatous skin biopsy specimens}

Paraffin blocks of skin biopsy specimens from eight patients with cutaneous manifestations of tuberculosis and six patients with cutaneous sarcoidosis were drawn from the pathology files from 1987 to 1993 at St Mary's Hospital. Biopsy specimens were selected because they exhibited extensive granuloma formation and were in a suitable condition to allow immunohistochemical procedures. The specimens were stained with antibodies for IL- $1 \beta$, fibronectin, $\alpha$-smooth muscle actin, TGF- $\beta$, and type 1 procollagen. (Staining for TNF- $\propto$ and PDGF$B$ was not performed on these samples because these antibodies required frozen specimens.)

Heaf biopsy specimens

Thirty six healthy subjects (tuberculosis contacts or with a past history of tuberculosis) with strongly positive (grade 3-4) Heaf reactions and 12 healthy controls with Heaf grade 2-3 reactions recruited from the St Mary's Hospital Chest Clinic were studied. Each of the 36 subjects underwent a Heaf test intradermally into the volar surface of the left forearm (tuberculin PPD, $100000 \mathrm{U} / \mathrm{ml}$, Evans Medical Ltd, Horsham, Surrey, UK). The 12 controls received normal saline using the same method of injection. After a time interval of 1-14 days six groups of six patients each underwent two punch skin biopsies (3 $\mathrm{mm}$ diameter; Steifel Laboratories (UK) Ltd, Woodburn Green, Buckinghamshire, UK) from within the area of the site of injection on days $1,2,3,6,10$, or 14 after the Heaf test. Thus, six subjects underwent two biopsies at each time point. Biopsy specimens were taken from the two groups of six controls on either day 3 or 14 . These procedures were performed under sterile conditions using subcutaneous local anaesthesia ( $1 \mathrm{ml} \mathrm{1 \%}$ lignocaine in adrenaline 1 in 100000 ). One biopsy specimen was fixed in $10 \%$ phosphate buffered formal saline and embedded in paraffin wax within 24 hours. Sections (5-6 $\mu \mathrm{m}$ thick) were cut for immunohistochemical examination with antibodies to type 1 procollagen (carboxy terminal domain), fibronectin, IL- $1 \alpha$, and $\alpha$-smooth muscle actin. The other biopsy specimen was snap frozen in a bath of liquid nitrogen at $-70^{\circ} \mathrm{C}$ in the preservative Cryo-M-bed (Bright Instruments Ltd, Huntingdon, Cambridgeshire, UK). Sections (3-4 $\mu \mathrm{m}$ thick) were cut on a cryostat for immunohistochemical examination with antibodies to TNF- $\alpha$ and PDGF-B.

All subjects gave their informed consent and the study was approved by the Parkside Area Health Authority ethics committee.

\section{IMMUNOCHEMICAL EXAMINATION}

Sections from each paraffin wax biopsy specimen were dewaxed, rehydrated, and treated with 3\% hydrogen peroxide in methanol to quench the activity of endogenous peroxidase. The sections were washed in phosphate buffered saline (PBS, $\mathrm{pH} 7.2$ ) and trypsinised (0.5\% trypsin and $0.5 \%$ chymotrypsin, Sigma Chemical, Poole, Dorset, UK) for 10 minutes for type 1 procollagen, IL- $1 \beta$, and $\alpha$-smooth muscle actin and 20 minutes for fibronectin. Non-specific protein binding was blocked with 1:5 normal porcine or rabbit serum (Dako Ltd, High Wycombe, Buckinghamshire, UK) in PBS. Frozen biopsy specimens were air dried for 10 minutes, fixed in acetone for 10 minutes, and washed in PBS for five minutes. The primary antibodies used in this study were monoclonal anti-human recombinant TNF- $\alpha$ (TCS Biologicals Ltd, Buckinghamshire, UK), rabbit anti-human IL-1 $\beta$ and rabbit anti-human PDGF-BB (Genzyme, Cambridge, Massachusetts, USA), rabbit anti-human panTGF- $\beta$ (R\&D, Abingdon, Oxon, UK), monoclonal mouse anti-human muscle actin (Dako Ltd), rabbit anti-human fibronectin (Sigma Chemical), and rabbit anti-human type 1 procollagen. This antibody is raised against the carboxy terminal domain of procollagen type I peptide which is cleaved enzymatically during the formation of collagen I and therefore detects cells actively synthesising collagen ${ }^{27}$ (gift from M J Warburton, St George's Hospital, London, $\left.\mathrm{UK}^{28}\right)$.

The antibodies were diluted in 1:20 porcine or rabbit serum in PBS. The sections were incubated with the optimal dilution of the primary antibodies for one hour in a humidifying chamber at room temperature (1:400 for type 1 procollagen, $1: 200$ for $\alpha$-smooth muscle actin and fibronectin) or overnight (1:100 for IL-1 $\beta$, PDGF-B, and TNF- $\alpha$ ). The sections were all washed with PBS and incubated with biotinylated secondary antibodies (Dako Ltd) for 30 minutes, washed, and then incubated with avidin biotin peroxidase complex (Dako Ltd), washed for 15 minutes, and visualised with 3 , 3'-diaminobenzidine (DAB) substrate (Dako 
Ltd). The sections were counterstained with Mayer's haematoxylin solution (Sigma Diagnostics, St Louis, USA) for one minute.

\section{CELL QUANTIFICATION}

After colour development slides from the Heaf biopsy specimens were scored in a coded fashion by a blinded observer as follows. Slides of paraffin wax sections (stained for type 1 procollagen, IL-1 $\beta, \alpha$-smooth muscle actin, and fibronectin) were scored by counting the numbers of positively staining nucleated cells, expressed as number $/ \mathrm{mm}^{2}$ grid (Graticules Ltd, Tunbridge, Kent, UK), at three levels of dermis (superficial, middle and deep) from both perivascular infiltrates and interstitial dermis infiltrates (areas of dermis selected not associated with vessels or sweat glands) and the mean calculated for each. Direct counting of cells in the frozen sections was not possible as their quality was not as good as the paraffin sections so each slide (stained for TNF- $\alpha$ or PDGF-B) was given an intensity score on a semiquantitative scale. Counting and grading of slides was performed by an observer blind to the code of the study. Specificity was ascertained when the primary antibody step was omitted or sections were treated with purified non-immune rabbit or mouse serum (Dako Ltd).

\section{STATISTICAL ANALYSIS}

Results are expressed as mean (SE). The 95\% confidence intervals (CI) for the difference between the two means (tuberculin Heaf test against control for days 3 and 14) were calculated using the confidence interval analysis programme (British Medical Journal, London, $\mathrm{UK})$.

\section{Results}

TUBERCULOSIS GRANULOMA BIOPSY SPECIMENS There was evidence of active synthesis of collagen both inside and outside the granulomas of all biopsy specimens examined. Numerous cells with fibroblast morphology staining positively for type 1 procollagen were seen scattered throughout the granuloma core and there was matrix staining around the margins of the granulomas which served to delineate one granuloma from another (fig $1 \mathrm{a}$ and $\mathrm{b}$ ). In addition, there was marked staining with antibodies to TGF- $\beta$ and fibronectin both in fibroblasts and in the extracellular matrix surrounding the granulomas, but not inside the granulomas themselves (fig $2 c, d$ and $f$ ). Only faint staining for IL-1 $\beta$ was seen, with occasional positively stained giant and epithelioid cells at the periphery of the granuloma. Epidermal staining for IL-1 $\beta$ was present in all specimens. No convincing positive staining for $\alpha$-smooth muscle actin was seen either in or around the granulomas. In all cases dense staining for $\alpha$-smooth muscle actin was present in vascular smooth muscle, serving as a positive control.
SARCOIDOSIS GRANULOMA BIOPSY SPECIMENS By contrast, extensive staining for fibronectin and TGF- $\beta$ was detected throughout the granulomas of all six sarcoidosis biopsy specimens with accentuation of cellular and matrix staining around the periphery of the granulomas (fig $2 a, b$ and $e$ ). The cells staining with antibody for TGF- $\beta$ appeared morphologically to be macrophages within the granulomas themselves, but fibroblasts within the surrounding rim. Cells staining for type 1 procollagen were less prominent than those in the tuberculosis sections, both inside and around the granulomas. However, the staining of the granuloma margins appeared more prominent (fig $1 \mathrm{c}$ and $\mathrm{d}$ ). Occasional staining with IL$1 \beta$ antibody was observed within cells at the margins of the granulomas. Again, there was little staining for $\alpha$-smooth muscle actin in the granulomas of these biopsy specimens. Substitution of non-immune serum for each of the primary antibodies yielded no immunostaining in specimens of tuberculosis and sarcoidosis lesions, confirming the specificity of the antibodies used.

\section{HEAF BIOPSY SPECIMENS}

Characteristics of the delayed type

hypersensitivity reaction

All subjects who underwent the tuberculin Heaf test reacted with red induration and swelling at the site of injection from day 1 onwards, reaching a peak at day 5 . Sections stained with haematoxylin and eosin revealed a patchy perivascular interstitial dermal and periadnexal infiltration of mononuclear cells from day 1 onwards, the size and density growing to a maximum at day 14 although this was variable and it was not easy to distinguish one day's biopsy specimen from another. Subjects who received normal saline did not show any lasting visible reaction. Sections stained with haematoxylin and eosin revealed very little cellular infiltrate on either day 3 or 14 .

\section{Type 1 procollagen}

Cellular and matrix staining was modestly but significantly increased from day 1 onwards (fig 3d); $95 \% \mathrm{CI}$ for the difference between the mean Heaf and control perivascular infiltrate counts 3.17 to 14.60 on day 3 and 28.2 to 63.7 on day 14 . The number of positively staining cells increased to a maximum at day 14 in both perivascular and dermal infiltrates (fig $1 \mathrm{e}-\mathrm{g}$ ). These cells were morphologically fibroblasts. Control sections exhibited little cellular and no background staining (fig $1 \mathrm{~h}$ ). Sections stained with rat anti-human procollagen 1 (Chemicon International Inc, Harrow, UK) which detects the cleaved amino-terminal domain of type I procollagen revealed similar patterns of staining (results not shown).

Interleukin $1 \beta$

Immunostaining for IL-1 $\beta$ demonstrated strong expression from day 1 in both peri- 

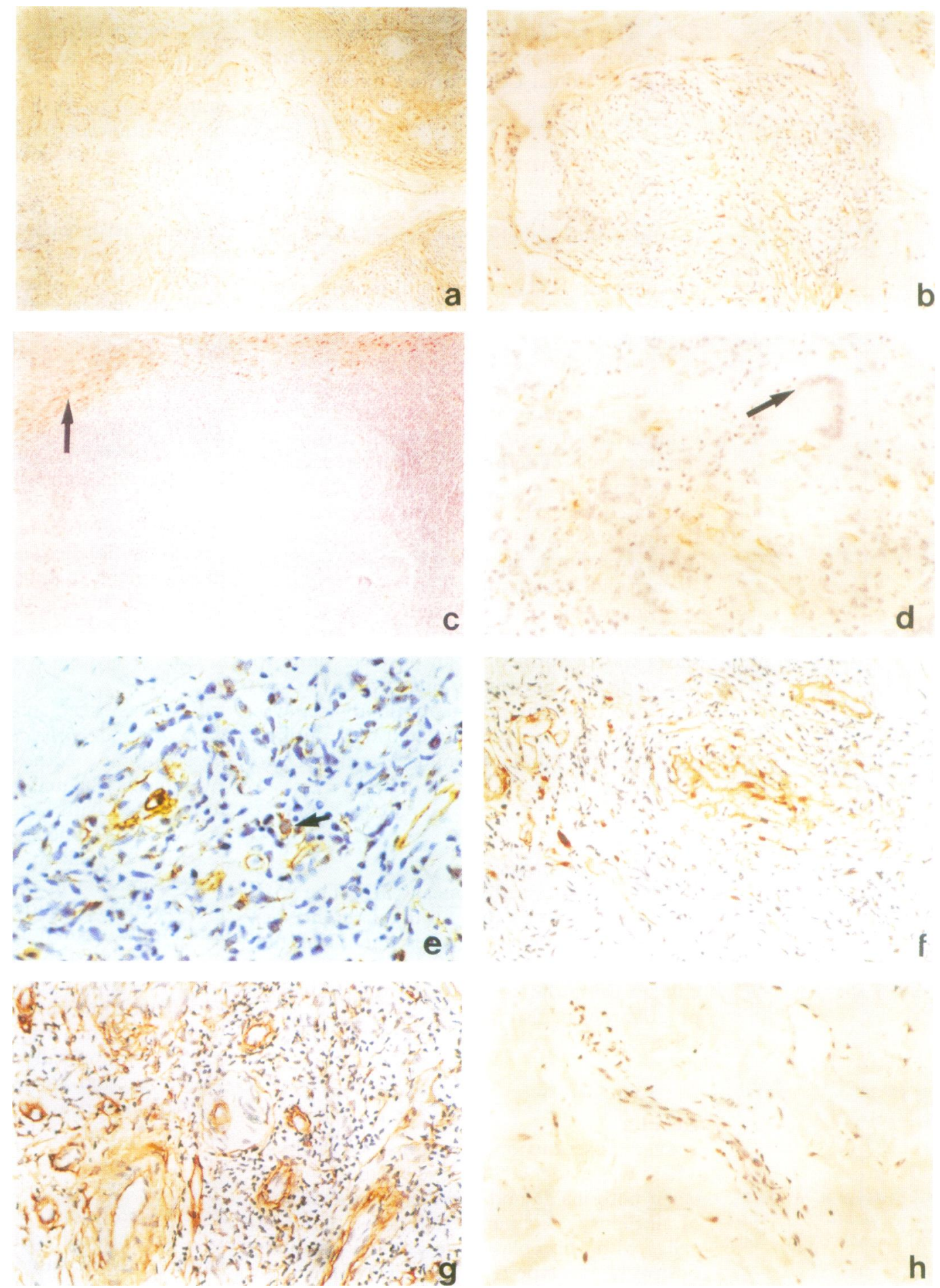

Figure 1 Photomicrographs of skin sections stained with anti-human type 1 procollagen antibody and counterstained with Mayer's haematoxylin solution demonstrating (a) cutaneous tuberculosis granulomas at $\times 50$ magnification and (b) at $\times 125$ magnification showing numerous positively staining cells in brown with highlighting of the granuloma

periphery, (c) cutaneous sarcoidosis granulomas at $\times 50$ magnification with less obvious cellular and matrix staining but prominent granuloma capsule staining (arrow) and $(d)$ at $\times 125$ magnification with multinucleate giant cell arrowed; and skin sections of tuberculin Heaf reactions stained with anti-human type 1 procollagen antibody and counterstained with Mayer's haematoxylin solution on $(e)$ day 3 after PPD inoculation $(\times 125$ magnification $)$ demonstrating negligible staining (arrow), $(f)$ day 10 after PPD inoculation ( $\times 125$ magnification), $(g)$ day 14 after PPD inoculation $(\times 125$ magnification), and (h) day 3 after inoculation with saline control.

vascular and dermal infiltrates which remained prominent in biopsy specimens collected throughout the 14 day study period (fig $3 a$ ). Cells staining positively were mainly found within cell aggregates (fig 4a). It was not possible to distinguish morphologically cell types staining with antibody. Absolute counts showed significantly increased numbers of positively stained cells compared with controls on days
3 and 14 (95\% CI for the difference between mean Heaf and control perivascular infiltrate counts 95.9 to 235 on day 3 and 38 to 146 on day 14) with up to one third of infiltrate cells staining. The pattern over the time course of the study was the same for the perivascular and dermal infiltrates. Only a few cells from the control biopsy specimens stained for IL- $1 \beta$ on days 3 and 14 . 

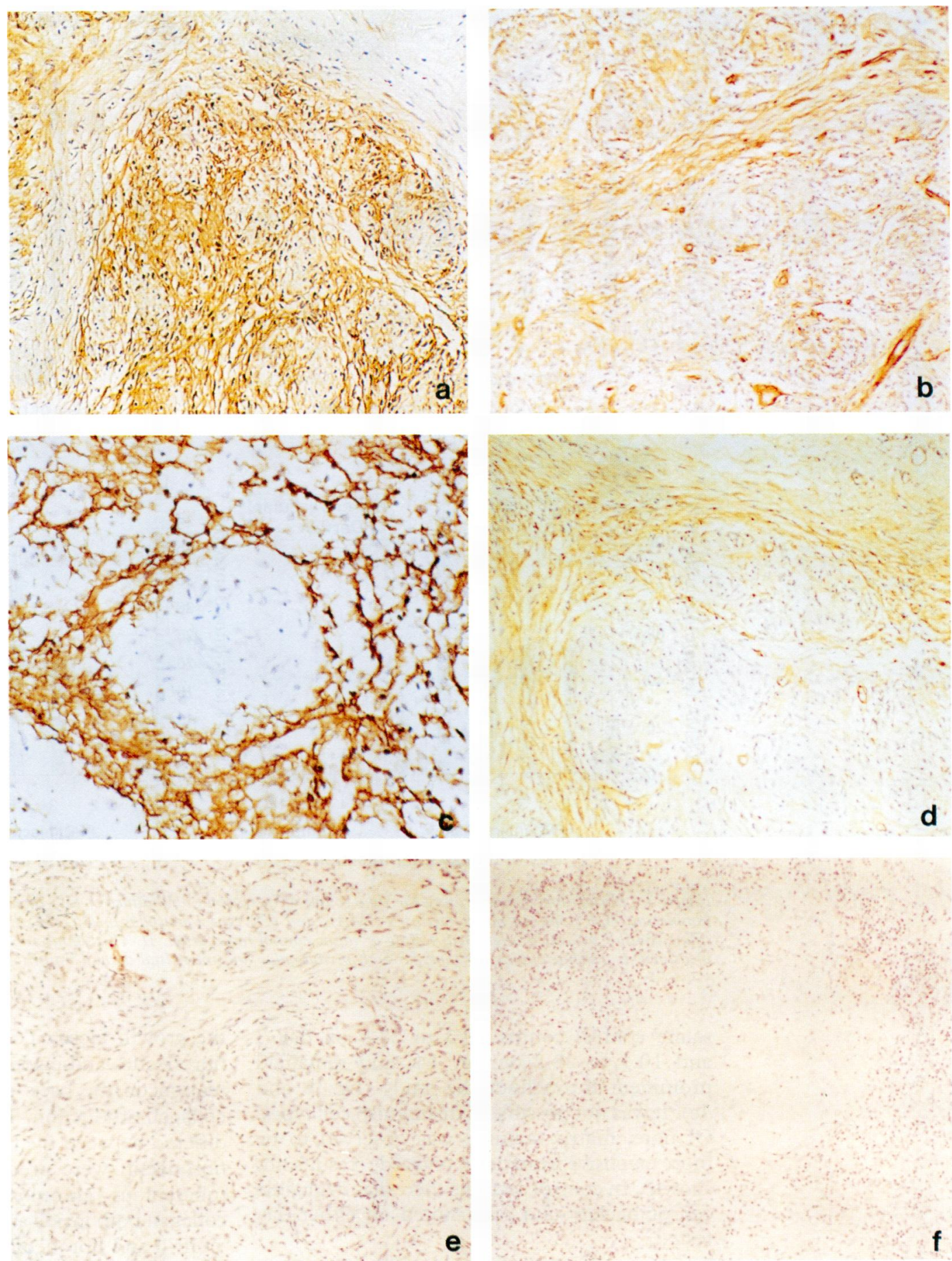

Figure 2 Photomicrographs of skin sections of cutaneous sarcoidosis granulomas stained with (a) anti-human transforming growth factor antibody and (b) anti-human fibronectin antibody demonstrating abundant staining throughout the granulomas, skin sections of cutaneous tuberculosis granulomas stained with (c) anti-human transforming growth factor and (d) anti-human fibronectin antibody demonstrating marked intracellular and extracellular staining in the granuloma rim but not within the granuloma, and sections of $(e)$ cutaneous sarcoidosis and $(f)$ tuberculosis granulomas with purified non-immune serum replacing primary antibody step omitted to confirm specificity of antibody staining.

Tumour necrosis factor $\alpha$

Abundant staining, most marked within the perivascular infiltrates in the skin biopsy specimens from tuberculin inoculated subjects, was observed from day 1 , peaking at day 3 (fig 4d), and remaining increased until day 7 . It was not possible to differentiate the cell types staining with antibody to TNF- $\alpha$.

Platelet derived growth factor $B$

There was increased cellular and matrix staining from day 1 which increased to a maximum

at day 3 (fig 4c), after which staining decreased to a moderate intensity (but still increased compared with controls) for the duration of the study. In the control sections only very mild staining or no staining was observed. It was possible to delineate the pattern of staining and how it related to the cellular infiltrate, but not to determine the morphology of the cells staining.

\section{Fibronectin}

Cell staining was increased from day 1 (95\% CI for the difference between mean Heaf and 


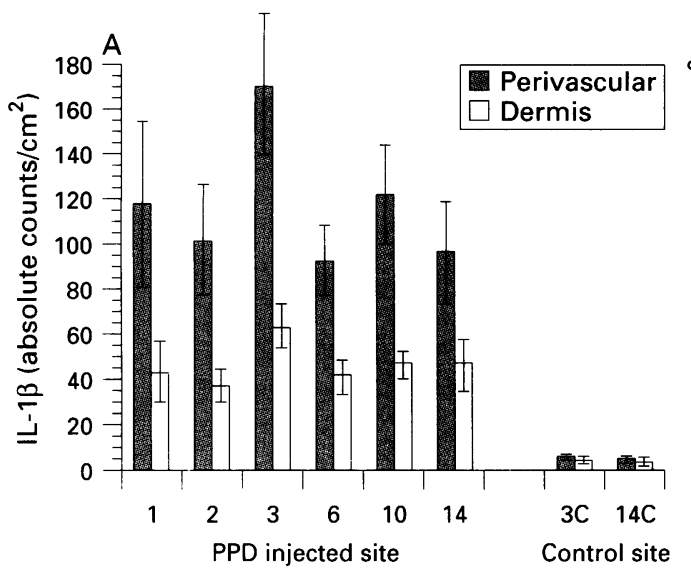

Day of biopsy after injection

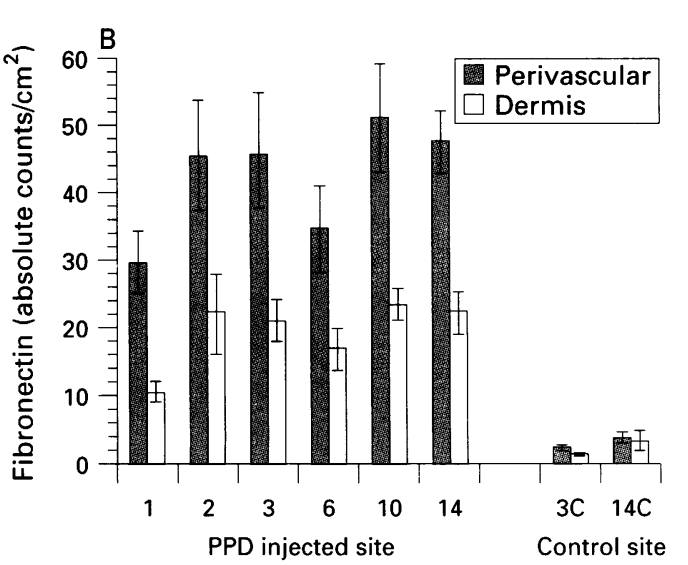

Day of biopsy after injection
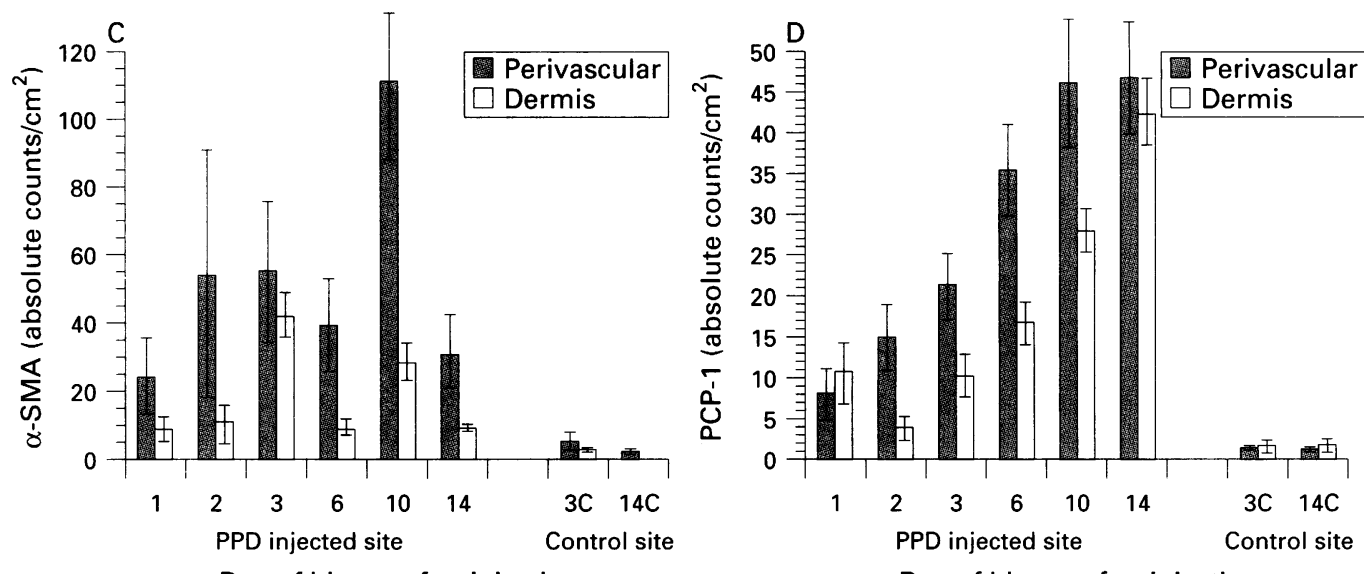

Day of biopsy after injection

Figure 3 Histograms demonstrating the time course of increase in abundance of $(A)$ interleukin $1 \beta(I L-1 \beta),(B)$ fibronectin, $(C) \alpha$-smooth muscle actin $(\alpha-S M A)$, and (D) type 1 procollagen (PCP-1) in Heaf biopsy specimens and saline controls measured after Heaf testing or saline control inoculation. IL-1 $\beta$, fibronectin, $\alpha-S M A$, and PCP-1 staining measured as absolute counts per $\mathrm{mm}^{2}$ for both perivascular and interstitial dermal infiltrates.

saline control counts 10.3 to 28.8 on day 3 and 10.1 to 28.2 on day 14 ) and remained prominent throughout the study (fig $3 \mathrm{~b}$ and 4e). It was not possible to distinguish cell types. Of note, matrix staining was observed to be more extensive from day 6 onwards (results not shown). In control sections there was negligible cell or background stain noted on either day tested.

\section{$\alpha$-smooth muscle actin}

Staining was increased from day 1 , rising to a peak on day 10 (figs $3 c$ and $4 b$ ) and remaining increased compared with control counts throughout the study ( $95 \%$ CI for the difference between the mean Heaf and control perivascular infiltrate counts 2.52 to 97.80 for day 3 and 3.47 to 54.1 for day 14). As before, the staining of the dermal infiltrates reflected that of the perivascular infiltrate and again there was little or no staining in the control biopsy specimens. Staining of vascular smooth muscle served as a positive control.

\section{Discussion}

These results indicate that granulomas from patients with skin involvement by tuberculosis and sarcoidosis exhibit increased expression of the growth factor TGF- $\beta$, the matrix protein fibronectin, and evidence of new collagen formation on the basis of increased type 1 procollagen production. The pattern of expression differed between the two diseases. TGF- $\beta$ and fibronectin were predominantly seen around tuberculous granulomas with type 1 procollagen both inside and around them, whereas TGF- $\beta$ and fibronectin was found throughout sarcoidosis granulomas with type 1 procollagen present but in smaller quantities within and surrounding the granulomatous tissue.

Excess accumulation of TGF- $\beta$ has been reported in granulomatous lung lesions in patients with sarcoidosis ${ }^{18}$ and tuberculosis. ${ }^{19}$ The present study extended these observations to the skin. Although the antibody used in the present study was a pan-TGF- $\beta$ antibody and thus did not provide information on TGF$\beta$ isoforms, the increase in TGF- $\beta$ was very marked. The consequences of excess TGF- $\beta$ production by these granulomas may be to promote local fibrotic processes. The observation that TGF- $\beta$ enhances the intracellular growth of Mycobacterium tuberculosis in macrophages ${ }^{1929}$ and counteracts macrophage activation by other cytokines ${ }^{30}$ suggests that TGF- $\beta$ might be beneficial to the host only after killing of mycobacteria is complete. 

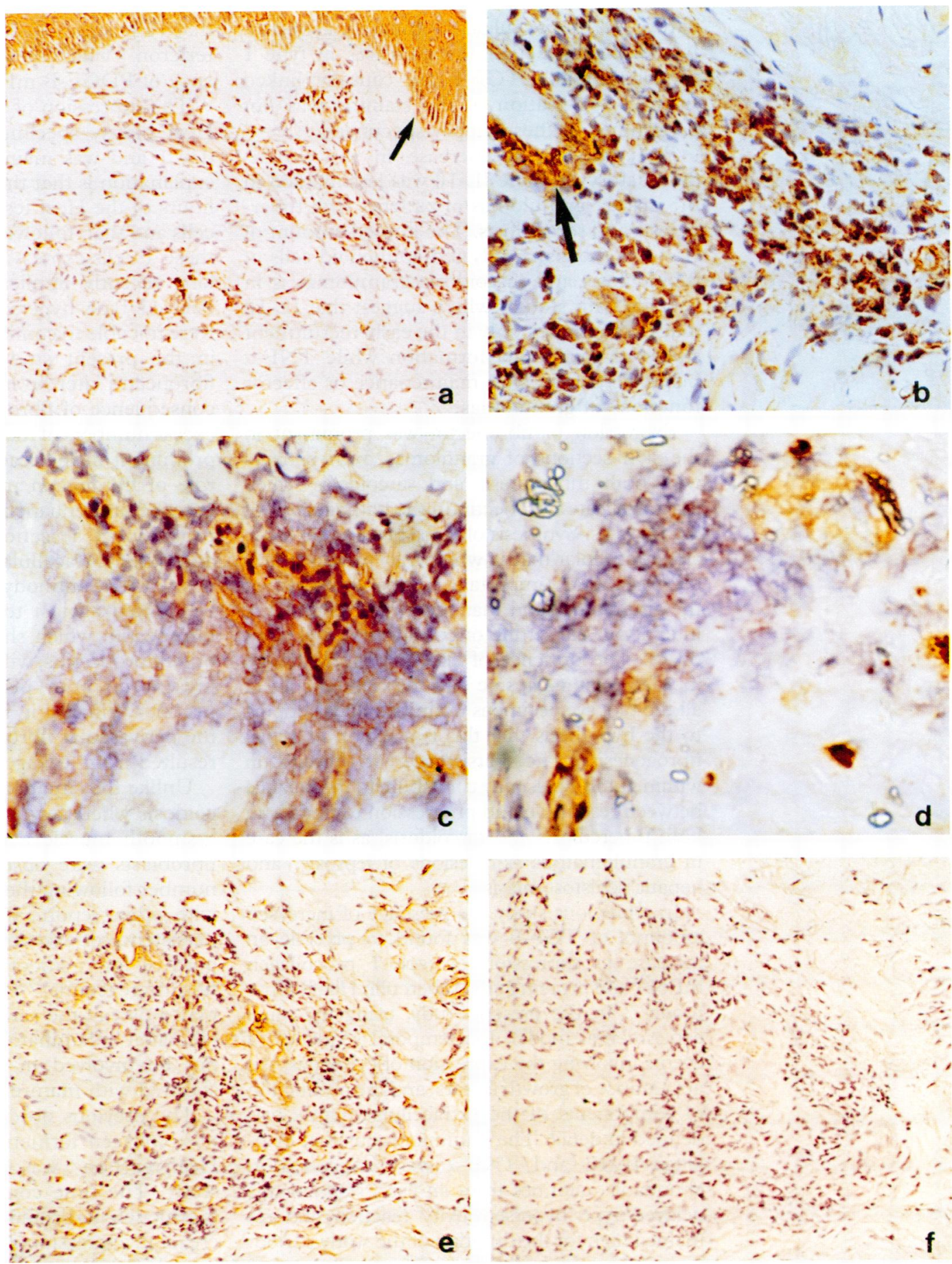

Figure 4 Photomicrographs of skin sections from biopsy specimens of tuberculin Heaf reactions six days after inoculation stained with antibodies against (a) interleukin-1 $\beta$ at $\times 100$ magnification (note strong positive stain of epidermis), (b) $\alpha$-smooth muscle actin at $\times 400$ magnification (note blood vessel wall staining positively, arrowed), (c) platelet derived growth factor $B$ (frozen section) at $\times 500$ magnification, (d) tumour necrosis factor $x$ at $\times 500$ magnification, (e) fibronectin at $\times 125$ magnification (note blood vessel wall staining positively) and $(f$ ) adjacent section to (e) with primary antibody replaced by non-immune serum.

Increased amounts of type 1 procollagen in granulomas of tuberculosis and sarcoidosis have not been reported previously. Antibodies to type 1 procollagen detect the terminal peptides of procollagen which are cleaved during the formation of mature collagen. ${ }^{27}$ The staining for type 1 procollagen, which was especially prominent in the tuberculous granulomas, therefore indicated that active production of new collagen was occurring. The observation that areas of extracellular matrix stained for type 1 procollagen may be the result of staining of newly cleaved carboxy terminal type 1 pro- collagen propeptide, since it is known that these cleavage reactions take place extracellularly, ${ }^{31}$ although these molecules are removed relatively quickly by extracellular endopeptidases. Alternatively, the staining that appears to be extracellular is, in fact, delicate strands of fibroblast cytoplasm. Lastly, this staining may be type 1 procollagen peptide since a small fraction of type 1 procollagen may escape complete conversion to collagen and be incorporated into the mature crosslinked collagen. ${ }^{32}$ These biochemical observations are in keeping with the recognised cicatrising 
nature of lupus vulgaris observed clinically. It is unclear whether the increases in type 1 procollagen and TGF- $\beta$ are causally linked since co-localisation of the staining was not determined in these biopsy specimens.

By contrast, the increase in the pro-inflammatory cytokine IL- $1 \beta$ was less marked in the mature granulomas of patients with tuberculosis and sarcoidosis. The limited amount of IL-1 $\beta$ in the giant cells and epithelioid cells of tuberculosis and sarcoidosis granulomas is in keeping with studies which suggest that IL-1 $\beta$ may be important only in the early recruitment stages of granuloma formation while TNF- $\alpha$ may take part in later maintenance or effector functions of granulomas. ${ }^{1133}$

Expression of fibronectin was prominent in the skin sections of granulomas from patients with both tuberculosis and sarcoidosis which may implicate fibronectin in the pathogenesis of granulomas. Synthesis of these proteins provides an extracellular matrix which may promote both cell migration (important in the evolution of the granuloma) and maintenance of granuloma integrity in the setting of considerable cell turnover. $^{34}$ The presence of excess amounts at the interface of the capsule and the cellular core in all the biopsy specimens examined adds support to the hypothesis that this is a region of active fibrogenesis. The persistence of fibronectin within granulomas may contribute to the balance between resolution and progression to fibrosis in both tuberculosis and sarcoidosis, as is the case in granulomatous skin lesions of leprosy ${ }^{35}$ and hepatic schistosomiasis. ${ }^{36}$

In a previous study the presence of increased amounts of TGF- $\beta$ was found to precede an increase in expression of type 1 procollagen mRNA and protein in the tuberculin Heaf biopsy model. ${ }^{37}$ In the present study, using the same experimental model, the temporal sequence of events involving the expression of cytokines, the appearance of growth factors, and the production of components of the extracellular matrix was defined in detail. The immunomodulatory cytokines IL- $1 \beta$ and TNF- $\alpha$ were expressed in abundant quantities from an early stage in this delayed type hypersensitivity reaction and remained high for the duration of the study period. This is in keeping with data from Chu et $a l^{26}$ who identified increases in IL-1 $\beta$ and TNF- $\alpha$ at high levels throughout a seven day study period following administration of PPD in a Mantoux test.

The present study did not repeat the earlier work in which TGF- $\beta$ was identified in the Heaf biopsy specimens ${ }^{37}$ but instead focused on the fibrogenic agent PDGF-B. Staining of frozen sections did not allow quantifiable data to be obtained but, by using a blinded scoring system, production of PDGF-B was found to be enhanced from as early as day 1 and reached a maximum well before the maximal expression of type 1 procollagen on day 14 (results not shown). The identification of this important growth factor for fibroblasts in the Heaf biopsy specimens may provide a mechanistic link between the delayed type hypersensitivity response and the first stages of a fibrotic reaction, and is in keeping with a previous observation that PPD stimulated lymphocytes produce interferon $\gamma$ which, in turn, increases the abundance of PDGF-B mRNA in macrophages. ${ }^{16}$

The expression of fibronectin by cells in the Heaf biopsy specimens occurred as early as day 1 and was maintained for 14 days. One explanation is that the cells that stained within hours of the Heaf test insertion are, in fact, $T$ cells and that, following the antigenic stimulus, these $T$ cells express $T$ cell fibronectin, a cytokine recently implicated in the initiation of delayed type hypersensitivity reactions. ${ }^{38} \mathrm{We}$ were not able to confirm this by double staining in the present study. The accumulation of fibronectin later on in the reaction might be a consequence of fibronectin production by the excess numbers of macrophages and fibroblasts prominent in the reaction at later stages. The role of fibronectin might now be in cell adherence and enhancing wound healing by the provision of a scaffold upon which collagen and other extracellular matrix proteins are deposited. The antibody used in the present study was raised against total fibronectin so $T$ cell fibronectin, which shares many epitopes with other fibronectins, will cross react with epitopes present on both plasma and cellular fibronectin. ${ }^{39}$ Finally, it is possible that some of the perivascular fibronectin staining may have resulted from leakage from the circulation.

Unlike the situation in tuberculosis and sarcoidosis where few cells staining positively for $\alpha$-smooth muscle actin were detected, myofibroblasts were observed to be increased in number following the Heaf test. This is in line with other reports which have indicated the persistence of excess numbers of cells staining positive for $\alpha$-smooth muscle actin in other fibrotic reactions. ${ }^{40}$ It is likely that these cells play a part in the early stages of a lymphocyte mediated inflammatory reaction, but they may not be involved in the fibrotic process associated with mature granulomas.

The previous observation of an increase in new collagen formation in association with a Heaf reaction ${ }^{37}$ was confirmed in the present study. The time course of the new collagen production indicated that it followed the increase in expression of inflammatory cytokines, growth factors including PDGF-B and TGF$\beta,{ }^{37}$ as well as $x$-smooth muscle actin and fibronectin. There is no clinical evidence that a Heaf test results in a permanent scar, so it is therefore likely that in the longer term there is remodelling and collagen removal. However, the time course in which a cellular influx is followed by an increase in cytokines and growth factors and later by evidence of new collagen production may serve as a useful human in vivo model in which to study the initiation of a fibrotic reaction during lymphocyte mediated inflammation. This may shed light on similar events that occur in tuberculosis and sarcoidosis granulomas and act as a human counterpart to animal studies to identify a link between the delayed type sensitivity reaction and fibrosis. ${ }^{41-44}$

A proposed scheme of the inflammatory and profibrotic events that occur within a tuberculosis or sarcoidosis granuloma resulting in 


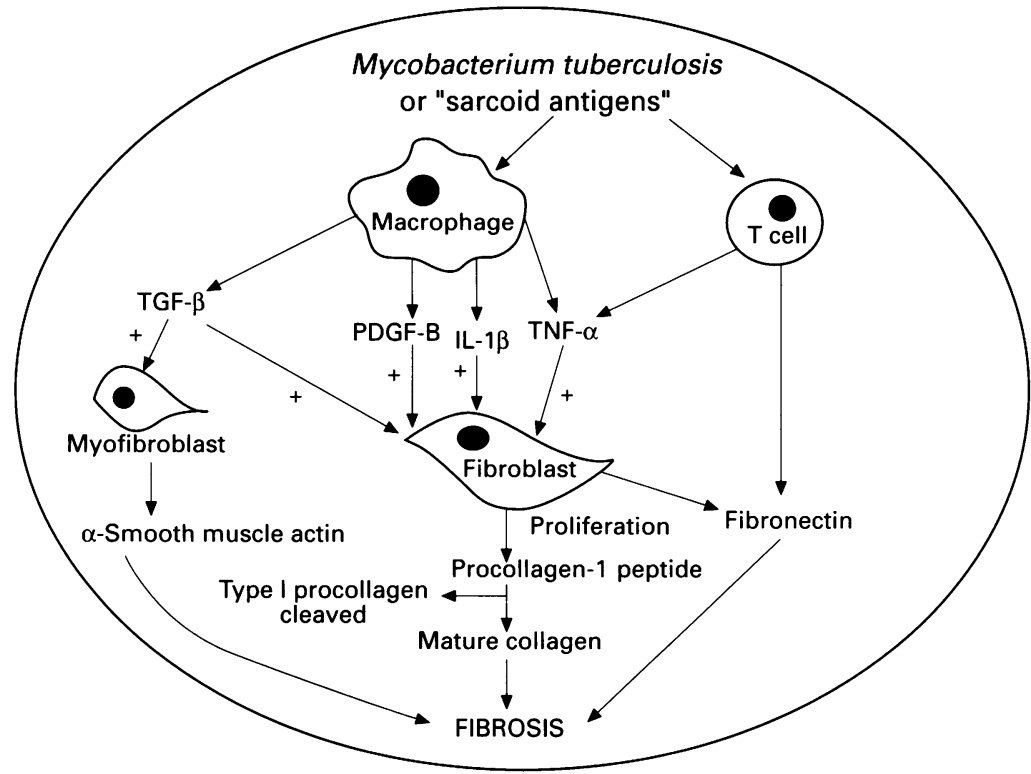

Figure 5 Diagram of proposed scheme of inflammatory and profibrotic events within a tuberculosis or sarcoidosis granuloma leading to excess deposition of extracellular matrix proteins and fibrosis. bacterium tuberculosis antigen (PPD)-stimulated lymphocytes. Clin Exp Immunol 1993;94:43-50.

17 Ishioka S, Yamakido M. Role of cytokines from BAL cells in granuloma formation. Nippon Rinsho 1994;52:1467-72.

18 Limper AH, Colby TV, Sanders M, Asakura S, Roch PC, DeRemee RA. Immunohistochemical localisation of transforming growth factor- $\beta 1$ in the non-necrotizing granulomas of pulmonary sarcoidosis. Am $\mathcal{f}$ Respir Crit Care Med 1994;149:197-204.

19 Toossi Z, Gogate P, Shiratsuchi H, Young T, Ellner JJ. Enhanced production of TGF- $\beta$ by blood monocytes from patients with active tuberculosis and presence of TGF- $\beta$ in tuberculous granulomatous lung lesions. $f$ Immunol in tuberculous gran

20 Kovacs EJ. Fibrogenic cytokines: the role of immune mediators in the development of scar tissue. Immunol Today 1991;12:17-23

21 Torikata C, Villiger B, Kuhn C, McDonald JA. Ultrastructural distribution of fibronectin in normal and fibrotic human lung. Lab Invest 1985;52:399-408.

22 Skalli O, Gabbiani G. The biology of the myofibroblast relationship to wound contraction and fibroconnective diseases. In: Clark RAF, Henson PM, eds. Molecular and cellular biology of wound repair. New York: Plenum, 1988: $373-401$.

23 Desmouliere A, Geinoz A, Gabbiani F, Gabbiani G. Transforming growth factor- $\beta$ induces $x$-smooth muscle actin expression in granulation tissue myofibroblasts and in quiescent and growing cultured fibroblasts. $\mathcal{f}$ Cell Biol quiescent and grow

24 Gibbs JH, Ferguson J, Brown RA, et al. Histometric study of the localisation of lymphocyte subsets and accessory cells in human Mantoux reactions. $\mathcal{F}$ Clin Pathol 1984;37: 1227-34.

25 Tsicopoulos A, Hamid Q, Varney V, et al. Preferential messenger RNA expression of Th1-type cells in classical delayed type (tuberculin) hypersensitivity reactions in human skin. f Immunol 1992;7:2058-61.

26 Chu CQ, Field M, Haskard D, Feldmann M, Maini RN. Detection of cytokines at the site of tuberculin-induced delayed-type hypersensitivity in man. Clin Exp Immunol 1990;90:522-9.

27 McDonald JA, Broekelmann TJ, Matheke ML, Crouch E, Koo M, Kuhn III C. A monoclonal antibody to the carboxyterminal domain of procollagen type I visualises callagen-synthesizing fibroblasts. $\mathcal{F}$ Clin Invest 1986;78: collagen-s

The authors thank Miss Sundhiya Mandalia for her assistance with the statistics and Professor Douglas Young for his help with the preparation of this manuscript. The study was funded by St Mary's Hospital Special Trustees and the British Lung Foundation.

1 Katz S. Clinical presentation and natural history of sarcoidosis. In: Fanberg BL, ed. Sarcoidosis and other granulomatous diseases of the lung. Switzerland: Marcel Dekker, 1983:3-36.

2 Savin JA. Sarcoidosis. In: Champion RH, Burton JL, Ebling FJG, eds. Textbook of dermatology. London: Blackwell Scientific, 1992:2383-406.

3 Quismorio Jr. FP. Immunological studies on cutaneous lesions in sarcoid. Clin Dermatol 1986;4:54-61.

4 Dannenberg Jr. AM. Roles of cytotoxic delayed-type hypersensitivity and macrophage-activating cell-mediated immunity in the pathogenesis of tuberculosis. Immunobiology 1994;191:461-73.

5 Hunninghake GW, Gadek JE, Young Jr RC, Kawanami $\mathrm{O}$, Ferrans VJ, Crystal RJ. Maintenance of granuloma formation in pulmonary sarcoidosis by T-lymphocytes within the lung. $N$ Engl $f$ Med 1980;302:594-8.

6 Dinarello CA. The biology of Interleukin-1. Interleukins. In: Kishimoto T, ed. Molecular biology and immunology. In: Kishimoto T, ed. Molecular
Switzerland: Karger, 1992:1-32.

7 Turino GM, Eden E. Interleukin-1 secretion from human alveolar macrophages in lung disease. $\mathcal{f}$ Clin Immunol 1986;6:326-33

8 Piguet PF, Collart MA, Grau GE, Kapanci Y, Vassalli P. Tumour necrosis factor/cachectin plays a key role in bleomycin-induced pneumopathy and fibrosis. $\mathcal{F} \operatorname{Exp} \mathrm{Med}$ 1989;170:655-63.

9 Piguet PF, Collart MA, Grau GE, Sappino A-P, Vassalli P. Requirement of tumour necrosis factor for development of silica-induced pulmonary fibrosis. Nature 1990;344: 245-7.

10 Kindler V, Sappino A-P, Grau GE, Piguet PF, Vassali P. The inducing role of tumour necrosis factor in the development of bacterial granulomas in BCG infection. Cell 1989;56:731-40.

11 Myatt N, Coghill G, Morrison K, Jones D, Cree IA. Detection of tumour necrosis factor alpha in sarcoidosis and tuberculosis granulomas using in situ hybridisation. $f$ Clin Pathol 1994;47:423-6.

12 Boros DL. The role of cytokines in the formation of the schistosome egg granuloma. Immunobiology 1994;191: 441-50.

13 Khalil N, O'Connor RN, Unruh HW, et al. Increased production and immunohistochemical localisation of transforming growth factor- $\beta$ in idiopathic pulmonary fibrosis. Am F Respir Cell Mol Biol 1991;5:155-62.

14 Wahl SM. Transforming growth factor $\beta$ : the good, the bad and the ugly. 7 Exp Med 1994;180:1587-90.

15 Shaw RJ, Benedict SH, Clark RAF, King Jr TE. Pathogenesis of pulmonary fibrosis in interstitial lung disease. Am Rev Respir Dis 1991;143:167-73.

16 Wangoo A, Taylor IK, Haynes AR, Shaw RJ. Up-regulation of alveolar macrophage platelet derived growth factor- $B$ (PDGF-B) mRNA by interferon gamma from Myco-
28 Warburton MJ, Ferns SA, Hughes C, Sear CH, Rudland PS. Generation of cell types with myoepithelial and mesenchymal phenotypes during the conversion of rat mammary tumor epithelial stem cells into elongated cells. $f$ Natl Cancer Inst 1987;78:1191-201.

29 Hirsch CS, Yoneda T, Averill L, Ellner JJ, Toossi Z. Enhancement of intracellular growth of Mycobacterium tuberculosis in human monocytes by transforming growth factorB. F Infect Dis 1994;170:1229-34.

30 Tsunawaki S, Sporn M, Ding A, Nathan C. Deactivation of macrophages by transforming growth factor- $\beta$. Nature of macrophages

31 Fessler JH, Fessler LI. Biosynthesis of procollagen. Ann Rev Biochem 1978;47:129-62.

32 Bornstein P, Sage H. Structurally distinct collagen types. Ann Rev Biochem 1980;49:974.

33 Remick DJ, Chensue SW, Hiserodt JC, Higashi GI, Kunkel SL. Flow-cytometric evaluation of lymphocyte subpopulations in synchronously developing Schistosoma mansoni egg and Sephadex bead pulmonary granulomas. $\mathrm{Am}$ $\mathcal{f}$ Pathol 1988;131:298-307.

34 Wangoo A, Cook HT, Taylor GM, Shaw RJ. Enhanced expression of type 1 procollagen and transforming growth factor- $\beta$ in tuberculin induced delayed type hypersensitivity. F Clin Pathol 1995;48:339-45.

35 Cree IA, Nurbai S, Milne G, Swanson-Beck J. Cell death in granulomata: the role of apoptosis. $\mathcal{F}$ Clin Pathol 1987; 40:1314-9.

36 Narayanan RB, Bhutani LK, Sharma AK, Nath I. Fibronectin in leprosy lesions: observations using monoclonal nectin in leprosy lesions: observations using monoclonal 532-9.

37 Grimaud J-A, Boros DL, Takiya C, Mathew RC, Emonard $H$. Collagen isotypes, laminin and fibronectin in granulomas of the liver and intestines of Schistosoma mansoniinfected mice. Am F Trop Med Hyg 1987;37:335-44.

38 Godfrey HP. T cell fibronectin: an unexpected inflammatory cytokine. Lymphokine Res 1990;9:435-47.

39 Godfrey HP, Canfield LS, Kindler HL, Angadi CV, Tomase $\mathrm{JJ}$, Goodman JW. Production of a fibronectin-associated lymphokine by cloned mouse T cells. F Immunol 1988; 141:1508

$40 \mathrm{McDonald}$ JA, Kuhn C. The roles of the myofibroblast in idiopathic pulmonary fibrosis. Ultrastructural and immunohistochemical features of sites of active extracellular matrix synthesis. Am f Pathol 1991;138:1257-65.

41 Miyamoto T, Kabe J, Noda M, Kobayashi N, Miura K. Physiological and pathological respiratory changes in delayed type hypersensitivity reaction in guinea pigs. $\mathrm{Am}$ Rev Respir Dis 1970;103:509-15.

42 Costabel U. The alveolitis of hypersensitivity pneumonitis. Eur Respir 7 1988;1:5-9.

43 Andrade ZA, Reed SG, Roters SB, Sadigursky M. Immunopathology of experimental cutaneous leishmaniasis. $A m^{\prime}$

44 Lemaire I. Selective differences in macrophage populations and monokine production in resolving pulmonary granuloma and fibrosis. Am $\mathcal{F}$ Pathol 1991;138:487-95. Pathol 1984;114:137-48. 